SHS Web of Conferences 21, 00002 (2015)

DOI: $10.1051 /$ shsconf/ 20152100002

C) Owned by the authors, published by EDP Sciences, 2015

\title{
Liste du comité scientifique
}

Sylvie Barma : Professeure agrégée

Faculté des sciences de l'éducation Département d'études sur l'enseignement et l'apprentissage Université Laval, Québec

Chercheure au CRIRES

sylvie.barma@fse.ulaval.ca

Marie-Claude Bernard : Professeure

CRIRES - Centre de recherche et d'intervention sur la réussite scolaire

Membre de l'ASIHVIF - Association internationale des histoires de vie en formation

Faculté des Sciences de l'éducation Québec, QC

marie-claude.bernard@fse.ulaval.ca

Catherine Boyer : MCF sciences de léducation- didactique des sciences

Laboratoire THEODILE- CIREL EA 4354 Université Charles De Gaulle- Lille3

Villeneuve d'Ascq

catherine.boyer@univ-lille3.fr

Maryline Coquidé : Professeure didactique des sciences

ENS Lyon - INRP

STEF Sciences Techniques Éducation Formation ENS de Cachan

maryline.coquide@ens-lyon.fr

Sandrine de Montgolfier : MC histoire des Sciences du Vivant

IRIS - CNRS/INSERM/EHESS/P13

Université Paris Est Créteil Val de Marne (UPEC), Espe de Créteil

sandrine.demontgolfier@u-pec.fr

This is an Open Access article distributed under the terms of the Creative Commons Attribution License 4.0, which permits unrestricted use, distribution, and reproduction in any medium, provided the original work is properly cited. 
Michèle Dell'Angelo : MC didactique des sciences / sciences de la vie et de la Terre STEF Sciences Techniques Éducation Formation ENS de Cachan

Université Paris Est Créteil (UPEC)

michele@dellangelo.com

Jacques Désautels : Professeur émérite, didacticien des sciences

Département d'études sur l'enseignement et l'apprentissage

Faculté des Sciences de l'éducation de l'Université Laval, Québec, QC jacques.desautels@fse.ulaval.ca

Magali Gallezot : MC didactique des Sciences

Laboratoire DidaScO-Université Paris Sud

gallezot@wanadoo.fr

Anne Sophie Godfroy : MC Philosophie

SND, Université Paris-Sorbonne-CNRS, FRE 3593

anne-sophie.godfroy@u-pec.fr

Michael Huchette : MC sciences et technologie

STEF Sciences Techniques Éducation Formation ENS de Cachan

Université Paris Est Créteil (UPEC), Espe de Créteil

michael.huchette@u-pec.fr

Frédéric Lalevée : Professeur-formateur de Sciences de la vie et de la Terre Lycée Jean Rostand, 93420 Villepinte-MAFPEN de l'académie de Créteil frederic.lalevee@ac-creteil.fr

Jean-Marc Lange : PU didactique des biosciences, de l'éducation à la santé, environnement et développement durable

Laboratoire CIVIIC (EA 2657) Université de Rouen

jean-marc.lange@univ-rouen.fr

Sébastien Lemerle : MC Sociologie

Université Paris Ouest Nanterre la Défense

Centre de recherches sociologiques et politiques de Paris

sebastien.lemerle@u-paris10.fr

Martine Paindorge: Maître de Conférences didactique des disciplines technologiques

Laboratoire Stef-ENS Cachan-Ifé

ESPE (École Supérieure du Professorat de l'Éducation) de Lorraine martine.paindorge@univ-lorraine.fr 
Camille Roux Goupille : MC en biologie Végétale / didactique des sciences STEF Sciences Techniques Éducation Formation ENS de Cachan Université Paris Est Créteil (UPEC) croux@u-pec.fr

Ghislain Samson : Professeur

Département des sciences de l'éducation

Université du Québec à Trois-Rivières

Ghislain.Samson@uqtr.ca

Catherine Simard : Professeure invitée en didactique des sciences et technologie Université du Québec à Rimouski,

Rimouski, Québec, Canada

Catherine_Simard@uqar.ca

Laurence Simonneaux : Professeur Didactique des Questions Socialement Vives, UMR EFTS ENFA université de Toulouse laurence.simonneaux@educagri.fr

\section{Remerciements}

Nous remercions toutes les personnes qui nous ont soutenu dans l'organisation du colloque puis dans la réalisation de cet ouvrage fruit étendu des travaux du colloque. Nous remercions ainsi tous les membres du comité scientifique qui ont participé aux travaux d'évaluation des contributions par une double lecture. Nous remercions également Manon Albert et Stéphanie Roux, étudiantes graduées en Sciences de l'éducation à l'Université Laval, pour leur soutien aux révisions de style de tous les articles.

Nous remercions tout particulièrement tous les partenaires institutionnels qui nous ont soutenu financièrement dans ce projet : l'institut de recherche interdisciplinaire sur les enjeux sociaux (IRIS), l'université Paris Est Créteil (UPEC), UMR STEF, du Centre de recherche et d'intervention sur la réussite scolaire (CRIRES), et l'université du Québec à Rimouski (UQAR). 


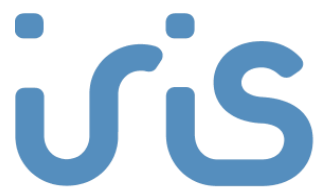

INSTITUT DE RECHERCHE INTERDISCIPLINAIRE SUR LES ENJEUX SOCIAUX SCIENCES SOCIALES, POLITIQUE, SANTÉ

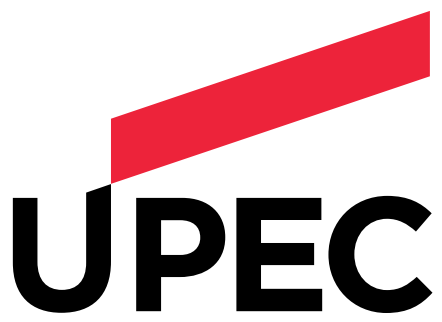

École supérieure D du professorat $\checkmark \int$ et de l'éducation UNIVERSITÉ PARIS-EST CRÉTEIL Académie de Créteil

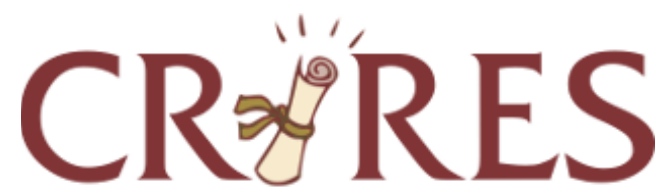

Centre de recherche et dintervention sur la réussite scolaire

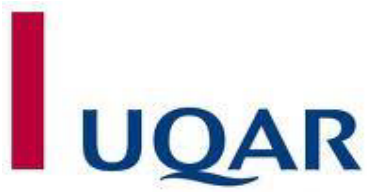

Université du Québec à Rimouski
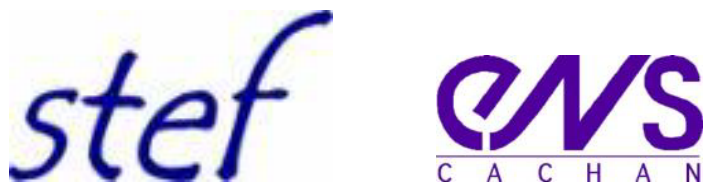